Schulich School of Law, Dalhousie University

Schulich Law Scholars

Research Papers, Working Papers, Conference

Papers

Faculty Scholarship

7-12-2012

\title{
Safety First: Recognizing and Managing the Risks to Child Participants in Magnetic Resonance Imaging Research
}

Jocelyn Downie

Dalhousie University - Schulich School of Law, jocelyn.downie@dal.ca

Matthias H. Schmidt

Dalhousie University, mhschmid@dal.ca

Follow this and additional works at: https://digitalcommons.schulichlaw.dal.ca/working_papers

\section{Recommended Citation}

Downie, Jocelyn and Schmidt, Matthias H., "Safety First: Recognizing and Managing the Risks to Child Participants in Magnetic Resonance Imaging Research" (2012). Research Papers, Working Papers, Conference Papers. 37.

https://digitalcommons.schulichlaw.dal.ca/working_papers/37

This Working Paper is brought to you for free and open access by the Faculty Scholarship at Schulich Law Scholars. It has been accepted for inclusion in Research Papers, Working Papers, Conference Papers by an authorized administrator of Schulich Law Scholars. For more information, please contact hannah.steeves@dal.ca. 


\title{
SAFETY FIRST: RECOGNIZING AND MANAGING THE RISKS TO CHILD PARTICIPANTS IN MAGNETIC RESONANCE IMAGING RESEARCH
}

\author{
MATTHIAS H. SCHMIDT ${ }^{1}$ and JOCELYN DOWNIE ${ }^{2}$ \\ ${ }^{1}$ Department of Radiology, Faculty of Medicine, Dalhousie University, Halifax, Nova Scotia, \\ Canada \\ ${ }^{2}$ Faculties of Law and Medicine, Dalhousie University, Halifax, Nova Scotia, Canada
}

Specialized and up-to-date knowledge is required to identify and manage the risks associated with advanced biomedical research. Additional complexities need to be considered when the research involves infants or young children. In this article, we focus on recent information about the physical risks of pediatric magnetic resonance imaging research and highlight information gaps. With an eye to assisting institutional review boards and researchers, we consider strategies for the management of these risks and formulate key questions aimed at exposing hidden hazards. Institutional review boards should ask these questions, and researchers should bear them in mind as they develop research protocols.

Keywords: bioethics, child, ethics, magnetic resonance imaging, research, safety, safety management

\section{Introduction}

Magnetic resonance imaging (MRI) is used widely to study the structure and function of the human body in vivo. MRI does not expose research participants to ionizing radiation, unlike imaging techniques based on x-ray transmission (e.g., computed tomography, CT) or radioisotope decay (e.g., positron emission tomography, PET). This makes MRI a particularly good imaging technique for serial studies and for studies involving children, who are more vulnerable to ionizing radiation than adults (Brenner et al., 2001).

However, MRI is not without hazard. As a result, a number of ethical concerns arise from MRI research. For example, what potential harms must be disclosed to prospective research participants or their surrogate decision-makers (Marshall et al., 2007)? How does the therapeutic misconception influence the informed consent process in MRI research (Hadskis et al., 2008)? Special concerns relating to child participants in MRI research are only beginning to be addressed (Downie and Marshall, 2007). For example, are there circumstances under which MRI research fails to meet the minimal risk standard, such that it would not be permissible to enroll children even with informed consent? What are the specific risks of pediatric MRI, and what can be done to manage these risks? Of all the important ethical issues surrounding pediatric MRI research, we believe that the issue of 
participant safety must receive priority. Therefore, we focus this paper on the physical hazards associated with pediatric MRI and on the management of risk in pediatric MRI research.

Recognizing the unique hazards to child participants in MRI research is the first and most important step in risk management. There are special complexities that need to be considered. For example, special technical and procedural adaptations may be required to image children safely and effectively. Risk management strategies must also take into account children's unique physiology and psychology and must include siblings, parents, and other caregivers who are an integral part of the "pediatric research environment." Finally, it must be noted that MRI is a rapidly evolving field. New hazards continue to be recognized. As is often the case with new technologies, the pediatric experience lags behind the adult experience. Safe pediatric MRI research, then, requires a very good understanding of children as well as a very good understanding of MRI. By synthesizing current information on the physical risks of pediatric MRI, highlighting information gaps, presenting both procedural and substantive strategies for risk management and formulating questions aimed at exposing hidden hazards, we hope to assist institutional review boards (IRBs) and researchers in their shared goal to ensure the safety of children participating in MRI research. ${ }^{1}$

\section{Fragile Research Participants}

It is a common adage in pediatrics that "children are not small adults." This is particularly true for infants, whose small size and immature homeostatic defenses make them uniquely vulnerable to perturbations in their environment. Imaging procedures that are considered entirely safe in adults and healthy older children cannot be assumed to be safe in this special population of patients. Yet, even preterm infants, the most fragile members of this population, are being recruited into MRI research studies (e.g., see Dudink et al. (2008) for a review of studies on normal myelination and white matter injury in preterm infants). ${ }^{2}$

Stokowski (2005) has recently reviewed the special safety issues pertaining to preterm infants and sick term infants who travel between a neonatal intensive care unit and an MRI scanner. ${ }^{3}$ These fragile patients require protective isolettes that maintain constant temperature, air flow, and humidity. They are often dependent on a large number of additional devices, such as endotracheal and enteric tubes, arterial and venous lines, temperature and oxygen saturation probes, urinary catheters, and electrocardiogram leads. Dislodgment of some of these devices during transport can be disastrous. Once in the scanner, temperature, heart rate, and oxygen saturation need to be monitored continuously. Ventilators and programmable infusion pumps, designed to deliver tiny

\footnotetext{
${ }^{1}$ While issues associated with the disclosure of risks to participants lie beyond the scope of this article, which focuses on risk identification and management, the questions listed at the end of each section could be used to guide the informed consent process between researchers and participants or their surrogate decision-makers. ${ }^{2}$ A search of ClinicalTrials.gov, using the search terms "neonate" and "MRI," revealed 31 registered trials that are currently recruiting healthy and sick neonates (http://clinicaltrials.gov/ct2/search. Accessed April 8, 2009.)

${ }^{3}$ Many of these concerns apply also to critically ill older children who are patients in intensive care units.
} 
volumes of fluid with high accuracy, must not be interrupted. Devices used for monitoring and support in the neonatal intensive care unit may not be MRI compatible. ${ }^{4}$ Conversely, MRI compatible devices designed for use with adults may not be appropriately configured for use with children, especially tiny infants. Resuscitation may be required at any time. This is difficult and potentially dangerous in the MRI environment. A large team of caregivers, including pediatricians, nurses, and respiratory technologists, need to be on hand, and all of them need to be familiar with the principles of MRI safety.

Institutions that routinely perform clinical and research imaging of neonates have adopted strategies for limiting the risks associated with transport and transfer to the MRI scanner, including the use of an MRI compatible incubator that can be inserted directly into the scanner (Erberich et al., 2003; Blüml et al., 2004; Whitby et al., 2004) and a dedicated MRI scanner sited in the neonatal intensive care unit to minimize transport (Battin et al., 1998). These strategies are expensive and are not available at all pediatric institutions. Moreover, they do not fully address the issues raised above. Nurses and respiratory technologists who regularly care for infants are often in the best position to say what is needed to monitor and support these fragile patients. Researchers must then consider whether or not this can be accomplished in the MRI environment. To avoid potentially disastrous oversights, it is extremely important to have a multidisciplinary team with experience in the clinical care of the prospective participants, as well as experience in MRI, involved in protocol development.

Questions that must be addressed when participants are preterm infants, sick term infants, or critically ill older children:

1. Did protocol development occur with input from all disciplines that normally participate in the care of the prospective participants, including representation from pediatrics, nursing, and respiratory technology?

2. Have all items needed for monitoring and support of vital functions been identified, and has the MRI compatibility of each item been established?

3. Are explicit strategies in place for ongoing monitoring and management of vital functions, and for potential resuscitation?

4. Have all caregivers who will accompany participants been familiarized with the principles of MRI safety?

5. Is dedicated equipment for neonatal imaging that reduces the risks of transport and transfer being used? If not, why not?

\section{Size Matters: Small Participants and Large Machines}

No matter how "noninvasive," all imaging involves the input of some form of energy that interacts with the body to produce an interpretable signal. Because of their immaturity and their small size, infants who undergo MRI are particularly vulnerable to 2 forms of energy involved in this procedure: acoustic energy (i.e., noise) and radiofrequency energy. Both

\footnotetext{
${ }^{4} \mathrm{An}$ MRI compatible device neither poses a physical hazard nor malfunctions in the MRI scanner.
} 
increase with the magnetic field strength, and both depend upon the type of imaging sequence that is used.

\section{Acoustic Energy}

Noise is a byproduct of rapidly switching magnetic gradients in the MRI scanner and can be severe. Concern about the effects of noise exposure is more serious for infants than older children (American Academy of Pediatrics, Committee on Environmental Health, 1997). Indeed, sudden, loud noises on the order of 80 decibel $(\mathrm{dB})$ can induce hypoxia in infants (Long et al., 1980). Early work at a field strength of 1.5 Tesla (T) found dramatic changes in heart rate due to MRI scanner noise (Philbin et al., 1996; Taber et al., 1998). This finding was especially worrisome for preterm infants, whose cardiac output is governed by heart rate (Philbin et al., 1996). It should be noted that the noise levels actually experienced by infants were not reported in these studies. Moreover, little or no hearing protection was used. By contrast, physiological parameters were shown to be stable by subsequent investigators who imaged infants at $1 \mathrm{~T}$ and measured noise levels between $67 \mathrm{~dB}$ and 72 $\mathrm{dB}$ (Battin et al., 1998). These noise levels, in fact, are less than those associated with finger tapping on an incubator or closing of metal cabinet doors under an incubator (American Academy of Pediatrics, Committee on Environmental Health, 1997).

High field MRI scanners (i.e., $\geq 3 \mathrm{~T}$ ) remain a problem. In one study, all sequences tested on a $4.7 \mathrm{~T}$ scanner exceeded $110 \mathrm{~dB}$, a level at which hearing is deemed to be at risk (Counter et al., 2000). However, the conscientious use of noise reduction strategies makes it possible to dampen noise levels to $80 \mathrm{~dB}$ in a $3 \mathrm{~T}$ scanner (Williams et al., 2007) and to less than 90 $\mathrm{dB}$ in a $4.7 \mathrm{~T}$ scanner (De Vita et al., 2006). These strategies include trimmed earplugs, miniaturized earmuffs, and sound barriers around coils and isolettes (Williams et al., 2007; De Vita et al., 2006). Still, these noise levels are at the tolerance limits for infants. What sound level is safe from the point of view of long term effects on hearing? Studies that correlate actual noise levels, age at exposure, and acuity of hearing at maturity are lacking. Any forthcoming studies are likely to be confounded by numerous uncontrollable and difficult-to-document environmental exposures. It is fair to say that no satisfactory answer exists.

Questions that must be addressed, especially when participants are infants:

1. Can the researchers keep the noise levels experienced by participants in the scanner below $80 \mathrm{~dB}$ ?

2. Are the researchers using all available means to reduce noise exposure?

\section{Radiofrequency Energy}

Radiofrequency energy deposition is necessary for the generation of images in MRI. It can have adverse effects, such as an increase in core body temperature, focal tissue heating, and even skin burns (Marshall et al., 2007). Radiofrequency energy deposition is expressed in terms of the specific absorption ratio (SAR), in units of Watt/kilogram (W/kg). The U.S. Food and Drug Administration (FDA) has stipulated significant risk thresholds for SAR as applied to the head and the whole body (U.S. Food and Drug Administration, Center for 
Devices and Radiological Health, 2003). MRI scanners operating under normal conditions are designed not to exceed these thresholds. However, when high field scanners or high energy imaging sequences (e.g., echoplanar imaging) are used, it is not difficult to approach significant risk thresholds for SAR in infants and young children, due to their small size (Rutherford et al., 2004). Moreover, it is possible to override SAR limits with scanners operating in "research mode." For any given imaging sequence, SAR can be calculated prior to imaging, provided the participant's weight is known. Thus, SAR can be predicted for a group of prospective participants based on an estimated range of their weights.

Questions that must be addressed, especially when participants are infants:

1. Can the researchers keep the SAR associated with their imaging protocol below the significant risk threshold set by the U.S. Food and Drug Administration?

2. Have the imaging parameters been optimized to yield useful data at the lowest possible SAR?

\section{ULTRAHIGH FIELD SCANNERS}

MRI signal increases with field strength, and this has prompted the development of ultrahigh field scanners, such as a scanner currently operating at $8 \mathrm{~T}$ (Kangarlu et al., 2004). An examination of healthy persons and patients ranging in age from 20-70 years found only minor and transient physiological effects associated with scanning at $8 \mathrm{~T}$ (Yang et al., 2006). No comparable pediatric data are available at this time. Currently, the significant risk threshold for field strength set by the FDA is $8 \mathrm{~T}$ for adults, children, and infants older than 1 month, and $4 \mathrm{~T}$ for infants less than 1 month of age (U.S. Food and Drug Administration, Center for Devices and Radiological Health, 2003). Already, there is enthusiasm for clinical imaging at 9.4 T (Radiological Society of North America, 2008). MRI compatibility of a wide range of devices will need to be established at these ultrahigh fields before they can be used in such scanners, and any observable physiological effects will need to be documented in pediatric patients. The lessons learned in conventional MRI scanners must be remembered. Safety for infants and young children cannot be assumed, based on adult data.

Questions that must be addressed prior to research imaging of infants and young children with ultrahigh field scanners:

1. Can the researchers keep noise levels and SAR within acceptable limits (see previous sections) at the ultrahigh field strength?

2. Can the researchers provide information that demonstrates a lack of adverse physiological effects on infants and young children who have undergone research scans or clinical scans in this MRI scanner or another MRI scanner of equal or higher field strength?

\section{Managing Physical Hazards in the Pediatric Research Environment}

The management of physical hazards involved in MRI poses a special challenge in the pediatric research environment. MRI scanners are powerful magnets. It is well known that the magnetic forces involved in MRI scanning can turn loose ferromagnetic objects into 
dangerous missiles and cause some implanted medical devices to malfunction (Marshall et al., 2007). However, knowledge of the magnetic forces at work in an MRI facility does not always translate into safe conduct around these forces. Unless specifically trained to do so, many people give little thought to the pens, paperclips, keys or coins they carry. Young children often collect small objects that adults would ignore or discard, stash them away in their pockets and soon forget them. The sometimes unpredictable behavior of children must be taken into account, as illustrated by the case report of a child with a runny nose, who was sent for an MRI scan and was found to have a metallic paperclip hidden away in his nasal passages (Rodolà and Anastasi, 2004).

In the pediatric research environment, researchers are also responsible for the safety of accompanying adults, siblings, and friends, as well as the child participants themselves. Other caregivers may be involved if participants are patients in a hospital. It is easy to lose track of a wandering sibling while preparing a child participant for an MRI scan. In our experience, the potential for chaos and for safety lapses increases with the number of persons in the MRI environment, especially when some of these persons are lively young children.

The few deaths and serious injuries that have occurred in and around MRI scanners to date are attributed to the use of inappropriate or outdated information and failure to follow safety guidelines (Shellock and Crues, 2004). These guidelines, with periodic updates, can be found in the radiological literature (Kanal et al., 2002; Kanal et al., 2004; Kanal et al., 2007; see also Shellock and Crues, 2002 and Kanal et al., 2003). They emphasize the importance of limiting access, rigorously screening and carefully supervising all persons who will be in or near the MRI scanner. This kind of rigor is particularly important in the pediatric environment.

Questions that must be addressed whenever MRI research is to be carried out in a pediatric setting:

1. Are the researchers prepared to manage the flow of children, their caregivers and attending personnel in the MRI environment?

2. Do the researchers possess sufficient knowledge about child behavior to ensure safety in the MRI environment?

\section{Sedation}

MRI requires absolute immobility for imaging sequences that last several minutes and confinement that may add up to an hour or longer. This combination of enforced immobility and confinement for lengthy periods exceeds the tolerance limits of many young children, who may simply be unable to refrain from fidgeting or may be downright distressed by the situation (Hallowell et al., 2008). Data is lost due to movement in as many as $50 \%$ of scans in the 6--7 year age group and $70 \%$ of scans in the 3--4 year age group (Amundsen et al., 2005). In clinical practice, sedation is used liberally to overcome this problem. This approach is acceptable when a child's health depends upon the examination, 
and the scan cannot be completed without sedation. The appropriateness of sedation solely for research purposes, however, remains far more controversial.

\section{Sedation Solely for Research Purposes}

A recent study of sedation in a research context examined 59 children aged 3-4 years and 49 children aged 6-7 years who were sedated with intravenous propofol for a longitudinal MRI research study (Amundsen et al., 2005). Complications were limited to partial airway obstruction (treated with insertion of an oral airway) in 2 children, bradycardia (selflimited) in 1 child, excessive salivation in 1 child and accidental disconnection of the intravenous tubing through which the propofol was being delivered in 1 child (Amundsen et al., 2005). These researchers concluded that propofol sedation for research is safe and effective with appropriate participant selection, supplemental oxygen delivery and supervision by an experienced pediatric anesthesiologist (Amundsen et al., 2005).

Studies of pediatric sedation in the clinical setting over the past decade, while confirming an acceptable level of safety for clinical purposes, are far less reassuring from a research ethics point of view. In the first place, the rate of complications during sedation varies considerably with the type and number of drugs used. For example, when complications during sedation with 2 orally administered drugs, pentobarbital and chloral hydrate, were compared in 1,316 infants, investigators found that the rate of hypoxia varied from .2-1.6\% (Mason et al., 2004a). In a subsequent study of 16,467 pediatric sedations with a variety of drugs, the overall rate of respiratory complications varied from $0 \%$ with the use of intravenous midazolam alone to $1.8 \%$ with the use of intravenous pentobarbital and fentanyl in combination (Sanborn et al., 2005).

The route of drug administration also affects the likelihood of complications during sedation. In a study of 2,146 infants sedated with either oral or intravenous pentobarbital, hypoxia occurred in .2\% of infants when the drug was given orally and .9\% when the drug was given intravenously (Mason et al., 2004b). A randomized, controlled trial of oral chloral hydrate versus intravenous pentobarbital in 70 children aged 2-12 years found much higher rates of complications: hypoxia occurred in $11 \%$ of children sedated with oral chloral hydrate and $17 \%$ of children sedated with intravenous pentobarbital (Malviya et al., 2004). Two of the children in this study experienced severe hypoxia, defined as blood oxygen saturation below 90\% (Malviya et al., 2004).

Not surprisingly, the rate of complications also varies significantly with the expertise and organization of the team providing the sedation. In all of the above studies, specially trained nurses were supervised by radiologists. In a study comparing complications before and after the institution of a dedicated pediatric sedation service led by pediatric emergency physicians and critical care specialists, hypoxia occurred in $8.8 \%$ of 2,148 sedations supervised by radiologists and $4.6 \%$ of sedations supervised by the pediatric emergency physicians and critical care specialists (King et al., 2006). It was also noted that there were 4 "code blue" calls to the radiology department during the 23 month period prior to the implementation of the dedicated sedation service and none in the 23 months following implementation of this service (King et al., 2006). It is important to note that 
when serious complications have occurred in the past, outcomes were worse in nonhospital settings than in hospitals, largely due to inadequate resuscitation (Coté et al., 2000).

Delayed complications, including vomiting, drowsiness, irritability and hyperactivity, can occur within 24-48 hours of sedation. In some of the above studies, such delayed complications were noted with overall frequencies ranging from .3-.6\% (Mason et al., 2004 b) to $1.2-1.3 \%$ (Mason et al., 2004a). However, other investigators found delayed complications to be much more frequent, with gastrointestinal complaints occurring in 18$37 \%$, restlessness and agitation in 23-29\%, and motor imbalance in $66-85 \%$ of children (Malviya et al., 2004).

The variability in the rates of complications noted by different authors, the potential seriousness of procedural complications and the high frequency of unpleasant postprocedural complications are noteworthy. Many IRBs and researchers, including ourselves, do not endorse pediatric MRI examinations under sedation solely for research purposes. The evidence argues particularly strongly against pediatric research MRI examinations under sedation carried out in adult hospitals or in free-standing research institutes that are not located within pediatric hospitals (Coté et al., 2000). Strategies to avoid sedation altogether have been proposed, including the use of guided imagery (Smart, 1997) and practice sessions in a scanner simulator (Hallowell et al., 2007). IRBs and researchers who are willing to consider sedation solely for research purposes should be aware that comprehensive guidelines for sedation have been developed by the American Society of Anesthesiologists (2002) and the American Academy of Pediatrics (1992, 2002, and 2006).

Questions that must be addressed if IRBs are willing to consider sedation solely for research purposes:

1. Are the drug and route of delivery chosen the safest available for the intended participants?

2. Is the MRI research facility equipped, and are the researchers qualified to provide pediatric sedation safely and in accordance with published guidelines?

3. Will participants be monitored appropriately, and is the MRI research facility equipped for effective resuscitation?

4. Will the researchers be giving participants' families clear, written information about post-sedation adverse events, including instructions for contacting a physician in the case of such an event?

\section{Combined Research and Clinical Imaging Under Sedation}

Many IRBs would not consider sedation solely for research purposes. However, they might well consider combining research imaging with clinical imaging under sedation. Under such circumstances, caution is still indicated when the same team is responsible for both research and clinical imaging. There is an understandable temptation to increase the depth of sedation in order to accommodate both research and clinical imaging. However, it is important to realize that it can be difficult to predict the depth of sedation reached by a 
particular individual with a particular drug regimen, and that the frequency of adverse events increases as sedation is increased to achieve a desired level of immobility (Dial et al., 2001).

Questions that must be addressed for combined research and clinical imaging under sedation:

1. Will clinical imaging be completed before research imaging begins and before sedation wears off?

2. Is the research imaging protocol tailored to ensure that it does not increase the risks of sedation (e.g., by significantly prolonging scans or imposing a more stringent requirement for immobility)?

3. Is the clinician responsible for sedation independent of the researchers? If not, have the researchers sought input from an independent expert in pediatric sedation during protocol development?

\section{Contrast Enhancement}

Intravenously administered contrast agents are commonly used in clinical imaging and occasionally also in research imaging. MRI contrast agents are compounds containing the rare earth element gadolinium. Contrast agents greatly improve the delineation of blood vessels and permit the examination of blood supply to tissues. Contrast agents also greatly improve the detection and characterization of lesions throughout the body. Ironically, MRI contrast agents were long considered a safe alternative to the more noxious and nephrotoxic CT contrast agents, particularly in patients at risk of kidney failure. This notion has changed drastically in recent years. The emerging story of MRI contrast agents and nephrogenic systemic fibrosis (NSF) highlights the need for up-to-date knowledge when evaluating MRI protocols.

\section{Nephrogenic Systemic Fibrosis}

NSF is a painful, disabling and potentially fatal condition, resulting from the abnormal deposition of collagen in skin, esophagus, heart, lung, kidneys, and bones (Martin, 2008). The condition occurs in patients with impaired renal function who have been exposed to gadolinium based contrast agents (Penfield, 2008). The first case of NSF was identified in 1997 and reported in the literature in 2000 (Cowper et al., 2000). There are now at least 200 reported cases of NSF (Steen and Schwenger, 2007), and the mortality rate for reported cases is as high as $28 \%$ in one series (Mendoza et al., 2006). NSF occurs in children as well as adults. Nine pediatric cases of NSF, ranging in age from 8-19 years, have been documented, and 2 of these children have died of complications of the disease (Penfield, 2008).

The association between NSF and gadolinium only began to emerge in 2006 (Grobner, 2006), but is now considered strong (Penfield, 2008). Indeed, only 8 cases without a documented history of gadolinium exposure have been reported to date, and lack of gadolinium exposure could not be guaranteed in any of these cases (Penfield, 2008). 
Gadolinium compounds with a linear chemical structure, prone to dissociation of the toxic gadolinium ion, present the highest risk. Thus, gadodiamide has been associated with the majority of cases of NSF to date, but gadoversetamide and gadopentetate dimeglumine are also associated with a significant number of cases (Martin, 2008; Mendichovszky et al., 2007; Penfield, 2008). Other risk factors, less consistently associated with NSF, include inflammation, thrombosis, acidosis, erythropoietin use, hepatorenal disease, and liver transplantation (Martin, 2008; Penfield, 2008).

Based on the limited available evidence, it is difficult to know the actual risk of developing NSF after gadolinium exposure in a predisposed individual. The prevalence of NSF after a dose of gadolinium in patients with renal failure is currently estimated to range from 1.55\% (Broome et al., 2007; Deo et al., 2007; Othersen et al., 2007). Multiple doses of gadolinium vs. a single dose of gadolinium increase the relative risk of developing NSF from 6.67 to 44.5 (Othersen et al., 2007). Risk estimates specific to the pediatric population are not yet available. Guidelines for clinical practice are provided by the FDA (U.S. Food and Drug Administration, Center for Drug Evaluation and Research, 2008). These guidelines caution against the use of gadolinium based contrast agents in patients with severe acute or chronic renal insufficiency and in patients with any degree of acute renal insufficiency in the setting of hepatorenal disease or liver transplant. It should be noted that these guidelines are being updated frequently as new information becomes available, and that they do not yet specifically address pediatric practice.

\section{Contrast Allergy}

Even children with normal renal function face risks from intravenous administration of MRI contrast agents, due to the possibility of a serious allergic reaction. Information about the level of risk in children has lagged behind comparable information for adults. Initial estimates of risk, based on large studies of adults, showed that life-threatening allergic reactions are extremely rare. In these studies, which reviewed between 9,000 and 30,000 contrast administrations over periods of 4-10 years, the incidence of serious reactions was $.01-.48 \%$, with smaller percentages reported in studies having larger sample sizes (Murphy et al., 1996; De Ridder et al., 2001; Li et al., 2006). Comparable data specific to the pediatric population have only recently become available. In a study that included 13,344 children, the rate of allergic reactions to gadolinium compounds was .04\% in children, compared to .07\% in adults (Dillman et al., 2007a). Only 1 of 6 allergic reactions in children was rated as severe (Dillman et al., 2007a). Thus, the risk of a serious allergic reaction in children is at least as low as that in adults, and perhaps even lower. By comparison, the risk of an allergic reaction to CT contrast agents in children ranges from .18\% (non-ionic contrast agents, Dillman et al., 2007b) to .4\% (ionic contrast agents, Gooding et al., 1975).

\section{Contrast Enhancement Solely for Research Purposes}

Similar to research involving sedation, the possibility of an acute, life-threatening contrast reaction mandates that any research involving contrast enhancement be performed in a hospital setting with the means and expertise required for pediatric resuscitation. For children with renal impairment, the use of gadolinium based contrast material solely for 
research purposes is out of the question. Infants, because of their immature renal function, should likewise not receive gadolinium based contrast agents solely for research purposes. It should be noted that contrast enhancement is not always necessary. The conspicuity of many lesions is excellent on unenhanced inversion recovery sequences. Arteries and veins can be studied with unenhanced time-of-flight sequences. Even tissue perfusion can be studied without contrast agents, using arterial spin labeling techniques.

Questions that must be addressed if REBs are willing to consider contrast enhancement solely for research purposes:

1. Can the researchers demonstrate that contrast enhancement is necessary to achieve the scientific goals of the research?

2. Do the researchers have a process to prevent children with risk factors for NSF (especially renal problems) from being enrolled in a study using a gadolinium compound?

3. Are the researchers using contrast material with the lowest probability of gadolinium dissociation, and hence the best theoretical safety profile with respect to NSF?

4. Are the researchers qualified and is the facility equipped to deal with lifethreatening allergic reactions?

5. Will the researchers be following up with participants and their families to identify delayed adverse events?

\section{Combined Contrast Enhanced Research and Clinical Imaging}

As with sedation, it might not be unreasonable to combine contrast enhanced research imaging with clinical imaging in the absence of contraindications. This is obviously true when plans have already been made for a contrast enhanced clinical examination. It may even be true when an unenhanced clinical examination was initially planned. Lundby et al. (1996) suggest that contrast enhancement improves the clinical information contained in MRI scans in 35-65\% of cases and uncovers entirely new information in $20-80 \%$ of cases. ${ }^{5}$

Questions that must be addressed when contrast enhanced research imaging and clinical imaging are combined:

1. Can the contrast enhanced research images be used clinically? If not, is the half-life of the contrast agent long enough to allow additional enhanced clinical images to be obtained?

2. Has the clinical value of enhancement been determined independently by a physician involved in the care of the participants?

\footnotetext{
${ }^{5}$ This study was conducted with involvement by manufacturers of the contrast agent.
} 


\section{Conclusions}

MRI is an invaluable technique for the study of anatomy and physiology in both healthy and diseased persons, and lack of ionizing radiation makes MRI especially attractive for pediatric research. However, there are definite hazards associated with the technique. Fortunately, most of these hazards can be controlled with forethought and a strong commitment to safety. In the ethics reviews of MRI research protocols, it is important for IRBs to keep the issue of participant safety front-and-centre. As a group, infants and young children require a particularly thoughtful examination of potential harms arising from MRI research. Some of these risks may not be obvious to MRI researchers who do not routinely work with children. Conversely, they may be overlooked or underestimated by pediatric researchers who are novices to MRI. A sound understanding of children's special physiology and psychology, as well as the unique aspects of the pediatric research environment, is needed. One cannot anticipate every possible hazard, but harm can be prevented or reduced by ensuring that: 1) protocols are developed, reviewed, and implemented by multidisciplinary teams of individuals with first-hand experience in pediatrics and the pediatric imaging environment; and 2) appropriate risk reduction strategies are employed.

\section{Acknowledgments}

The authors gratefully acknowledge the support of the Canadian Institutes for Health Research through a CIHR New Emerging Team Grant. The authors further wish to express their appreciation to Brad Abernethy, Matthew Kutcher, and members of the Neuroethics NET for helpful discussion and insightful comments.

\section{References}

American Academy of Pediatrics, Committee on Drugs. (1992). Guidelines for monitoring and management of pediatric patients during and after sedation for diagnostic and therapeutic procedures. Pediatrics, 89(6 Pt 1): 1110-1115.

American Academy of Pediatrics, Committee on Drugs. (2002). Guidelines for monitoring and management of pediatric patients during and after sedation for diagnostic and therapeutic procedures: Addendum. Pediatrics, 110(4): 836-838.

American Academy of Pediatrics, American Academy of Pediatric Dentistry, Coté, C. J., Wilson, S. and the Work Group on Sedation. (2006). Guidelines for monitoring and management of pediatric patients during and after sedation for diagnostic and therapeutic procedures: An update. Pediatrics, 118(6): 2587-2602.

American Academy of Pediatrics, Committee on Environmental Health. (1997). Noise: A hazard for the fetus and newborn. Pediatrics, 100(4): 724-727.

American Society of Anesthesiologists, Task Force on Sedation and Analgesia by NonAnesthesiologists. (2002). Practice guidelines for sedation and analgesia by nonanesthesiologists. Anesthesiology, 96(4): 1004-1017.

Amundsen, L. B., Artru, A. A., Dager, S. R., Shaw, D. W. W., Friedman, S., Sparks, B., and Dawson, G. (2005). Propofol sedation for longitudinal pediatric neuroimaging research. Journal of Neurosurgical Anesthesiology, 17(4): 180-192. 
Battin, M., Maalouf, E. F., Counsell, S., Herlihy, A., Hall, A., Azzopardi, D., and Edwards, A. D. (1998). Physiological stability of preterm infants during magnetic resonance imaging. Early Human Development, 52(2): 101-110.

Blüml, S., Friedlich, P., Erberich, S., Wood, J. C., Seri, I., and Nelson, M. D., Jr. (2004). MR imaging of newborns by using an MR-compatible incubator with integrated radiofrequency coils: Initial experience. Radiology, 231(2): 594-601.

Brenner, D. J., Elliston, C. D., Hall, E. J., and Berdon, W. E. (2001). Estimated risks of radiation-induced fatal cancer from pediatric CT. AJR American Journal of Roentgenology, 176(2): 289-296.

Broome, D. R., Girguis, M. S., Baron, P. W., Cottrell, A. C., Kjellin, I., and Kirk, G. A. (2007). Gadodiamide-associated nephrogenic systemic fibrosis: Why radiologists should be concerned. AJR American Journal of Roentgenology, 188(2): 586-592.

Coté, C. J., Notterman, D. A., Karl, H. W., Weinberg, J. A., and McCloskey, C. (2000). Adverse sedation events in pediatrics: A critical incident analysis of contributing factors. Pediatrics, 105(4 Pt 1): 805-814.

Counter, S. A., Olofsson, Å., Borg, E., Bjelke, B., Häggström, A., and Grahn, H. F. (2000). Analysis of magnetic resonance imaging acoustic noise generated by a $4.7 \mathrm{~T}$ experimental system. Acta Otolaryngologica, 120(6): 739-743.

Cowper, S. E., Robin, H. S., Steinberg, S. M., Su, L. D., Gupta, S., and LeBoit, P. E. (2000). Scleromyxoedema-like cutaneous diseases in renal-dialysis patients. The Lancet, 356(9234): 1000-1001.

De Ridder, F., De Maeseneer, M., Stadnik, T., Luypaert, R., and Osteaux, M. (2001). Severe adverse reactions with contrast agents for magnetic resonance: Clinical experience in 30000 MR examinations. JBR-BTR, 84(4): 150-152.

De Vita, E., Bainbridge, A., Cheong, J. L. Y., Hagmann, C., Lombard, R., Chong, W. K., Wyatt, J. S., Cady, E. B., Ordidge, R. J., and Robertson, N. J. (2006). Magnetic resonance imaging of neonatal encephalopathy at 4.7 Tesla: Initial experiences. Pediatrics, 118(6): e1812e1821.

Deo, A., Fogel, M., and Cowper, S. E. (2007). Nephrogenic systemic fibrosis: A population study examining the relationship of disease development to gadolinium exposure. Clinical Journal of the American Society of Nephrology, 2(2): 264-267.

Dial, S., Silver, P., Bock, K., and Sagy, M. (2001). Pediatric sedation for procedures titrated to a desired degree of immobility results in unpredictable depth of sedation. Pediatric Emergency Care, 17(6): 414-420.

Dillman, J. R., Ellis, J. H., Cohan, R. H., Strouse, P. J., and Jan, S. C. (2007a). Frequency and severity of acute allergic-like reactions to gadolinium-containing IV contrast media in children and adults. AJR American Journal of Roentgenology, 189(6): 1533-1538.

Dillman, J. R., Strouse, P. J., Ellis, J. H., Cohan, R. H., and Jan, S. C. (2007b). Incidence and severity of acute allergic-like reactions to IV nonionic iodinated contrast material in children. AJR American Journal of Roentgenology, 188(6): 1643-1647.

Downie, J. and Marshall, J. (2007). Pediatric neuroimaging ethics. Cambridge Quarterly of Healthcare Ethics, 16(2): 147-160.

Dudink, J., Kerr, J. L., Paterson, K., and Counsell, S. J. (2008). Connecting the developing preterm brain. Early Human Development, 84(12): 777-782. 
Erberich, S. G., Friedlich, P., Seri, I., Nelson, M. D., Jr., and Blüml, S. (2003). Functional MRI in neonates using neonatal head coil and MR compatible incubator. NeuroImage, 20(2): 683-692.

Gooding, C. A., Berdon, W. E., Brodeur, A. E., and Rowen, M. (1975). Adverse reactions to intravenous pyelography in children. AJR American Journal of Roentgenology, 123(4): 802-804.

Grobner, T. (2006). Gadolinium--A specific trigger for the development of nephrogenic fibrosing dermopathy and nephrogenic systemic fibrosis? Nephrology Dialysis Transplantation, 21(4): 1104-1108.

Hadskis, M., Kenny, N., Downie, J., Schmidt, M., and D’Arcy, R. (2008). The therapeutic misconception: A threat to valid parental consent for pediatric neuroimaging research. Accountability in Research, 15(3): 133-151.

Hallowell, L. M., Stewart, S. E., de Amorim e Silva, C. T., and Ditchfield, M. R. (2008). Reviewing the process of preparing children for MRI. Pediatric Radiology, 38(3): 271279.

Kanal, E., Borgstede, J. P., Barkovich, A. J., Bell, C., Bradley, W. G., Felmlee, J. P., Froelich, J. W., Kaminski, E. M., Keeler, E. K., Lester, J. W., Scoumis, E. A., Zaremba, L. A., and Zinninger, M. D. (2002). American College of Radiology White Paper on MR Safety. AJR American Journal of Roentgenology, 178(6): 1335-1347.

Kanal, E., Borgstede, J. P., Barkovich, A. J., Bell, C., Bradley, W. G., Felmlee, J. P., Froelich, J. W., Kaminski, E. M., Keeler, E. K., Lester, J. W., Scoumis, E. A., Zaremba, L. A., and Zinninger, M. D. (2003). ACR Blue Ribbon Panel response to the ACR commentary by Shellock and Crues on the ACR white paper on MR safety. AJR American Journal of Roentgenology, 180(1): 31-35.

Kanal, E., Borgstede, J. P., Barkovich, A. J., Bell, C., Bradley, W. G., Etheridge, S., Felmlee, J. P., Froelich, J. W., Hayden, J., Kaminski, E. M., Lester, J. D., Jr., Scoumis, E. A., Zaremba, L. A., and Zinninger, M. D. (2004). American College of Radiology White Paper on MR Safety: 2004 update and revisions. AJR American Journal of Roentgenology, 182(5): 1111-1114.

Kanal, E., Barkovich, A. J., Bell, C., Borgstede, J. P., Bradley, W. G., Jr., Froelich, J. W., Gilk, T., Gimbel, J. R., Gosbee, J., Kuhni-Kaminski, E., Lester, J. W., Jr., Nyenhuis, J., Parag, Y., Schaefer, D. J., Sebek-Scoumis, E. A., Weinreb, J., Zaremba, L. A., Wilcox, P., Lucey, L., and Sass, N. for the ACR Blue Ribbon Panel on MR Safety. (2007). ACR guidance document for safe MR practices: 2007. AJR American Journal of Roentgenology, 188(6): 14471474.

Kao, S. C., Adamson, S. D., Tatman, L. H., and Berbaum, K. S. A survey of postdischarge side effects of conscious sedation using chloral hydrate in pediatric CT and MR imaging. (1999). Pediatric Radiology, 29(4): 287-290.

Kangarlu, A., Baudendistel, K. T., Heverhagen, J. T., and Knopp, M. V. (2004). Klinische Hochund Ultrahochfeld-MR und ihre Wechselwirkung mit biologischen Systemen. Der Radiologe, 44(1): 19-30.

King, W. K., Stockwell, J. A., DeGuzman, M. A., Simon, H. K., and Khan, N. S. (2006). Evaluation of a pediatric-sedation service for common diagnostic procedures. Academic Emergency Medicine, 13(6): 673-676.

Li, A., Wong, C. S., Wong, M. K., Lee, C. M., and Au Yeung, M. C. (2006). Acute adverse reactions to magnetic resonance contrast media-gadolinium chelates. The British Journal of Radiology, 79(941): 368-371. 
Long, J. G., Lucey, J. F., and Philip, A. G. S. (1980). Noise and hypoxemia in the intensive care nursery. Pediatrics 65(1): 143-145.

Lundby, B., Gordon, P., and Hugo, F. (1996). MRI in children given gadodiamide injection: Safety and efficacy in CNS and body indications. European Journal of Radiology, 23(3): 190-196.

Malviya, S., Voepel-Lewis, T., Prochaska, G., and Tait, A. R. (2000). Prolonged recovery and delayed side effects of sedation for diagnostic imaging studies in children. Pediatrics, 105(3): E42.

Malviya, S., Voepel-Lewis, T., Tait, A. R., Reynolds, P. I., Gujar, S. K., Gebarski, S. S., and Eldevik, O. P. (2004). Pentobarbital vs chloral hydrate for sedation of children undergoing MRI: Efficacy and recovery characteristics. Pediatric Anesthesia, 14(7): 589595.

Marshall, J., Martin, T., Downie, J., and Malisza, K. (2007). A comprehensive analysis of MRI research risks: In support of full disclosure. The Canadian Journal of Neurological Sciences, 34(1): 11-17.

Martin, D. R. (2008). Nephrogenic systemic fibrosis. Pediatric Radiology, 38(Suppl 1): S125S129.

Mason, K. P., Sanborn, P., Zurakowski, D., Karian, V. E., Connor, L., Fontaine, P. J., and Burrows, P. E. (2004a). Superiority of pentobarbital versus chloral hydrate for sedation in infants during imaging. Radiology, 230(2): 537-542.

Mason, K. P., Zurakowski, D., Connor, L., Karian, V. E., Fontaine, P. J., Sanborn, P. A., and Burrows, P. E. (2004b). Infant sedation for MR imaging and CT: Oral versus intravenous pentobarbital. Radiology, 233(3): 723-728.

Mendichovszky, I. A., Marks, S. D., Simcock, C. M., and Olsen, Ø. E. (2008). Gadolinium and nephrogenic systemic fibrosis: Time to tighten practice. Pediatric Radiology, 38(5): 489-496.

Mendoza, F. A., Artlett, C. M., Sandorfi, N., Latinis, K., Piera-Velazquez, S., and Jimenez, S. A. (2006). Description of 12 cases of nephrogenic fibrosing dermopathy and review of the literature. Seminars in Arthritis and Rheumatism, 35(4): 238-249.

Murphy, K. J., Brunberg, J. A., and Cohan, R. H. (1996). Adverse reactions to gadolinium contrast media: A review of 36 cases. AJR American Journal of Roentgenology, 167(4): 847-849.

Othersen, J. B., Maize, J. C., Jr., Woolson, R. F., and Budisavljevic, M. N. (2007). Nephrogenic systemic fibrosis after exposure to gadolinium in patients with renal failure. Nephrology Dialysis Transplantation, 22(11): 3179-3185.

Penfield, J. G. (2008). Nephrogenic systemic fibrosis and the use of gadoliniumbased contrast agents. Pediatric Nephrology, 23(12): 2121-2129. Philbin, M. K., Taber, K. H., and Hayman, L. A. (1996). Preliminary report: Changes in vital signs of term newborns during MR. AJNR American Journal of Neuroradiology, 17(6): 1033-1036.

Radiological Society of North America. (2008). 9.4T promises greater function, new purpose for MR. RSNA News, 18(7): 6-7.

Rodolà, F. and Anastasi, F. (2004). A runny nose: An unexpected ferromagnetic foreign body in an unexpected place. European Review for Medical and Pharmacological Sciences, 8(4): 139-141. 
Sanborn, P. A., Michna, E., Zurakowski, D., Burrows, P. E., Fontaine, P. J., Connor, L., and Mason, K. P. (2005). Adverse cardiovascular and respiratory events during sedation of pediatric patients for imaging examinations. Radiology, 237(1): 288-294.

Shellock, F. G. and Crues, J. V., III. (2002). MR safety and the American College of Radiology White Paper. AJR American Journal of Roentgenology, 178(6): 1349-1352.

Shellock, F. G. and Crues, J. V. (2004). MR procedures: Biologic effects, safety, and patient care. Radiology, 232(3): 635-652.

Smart, G. (1997). Helping children relax during magnetic resonance imaging. MCN The American Journal of Maternal/Child Nursing, 22(5): 237-241.

Stokowski, L. A. (2005). Ensuring safety for infants undergoing magnetic resonance imaging. Advances in Neonatal Care, 5(1): 14-27.

Steen, H. and Schwenger, V. (2007). Good MRI images: To gad or not to gad? Pediatric Nephrology, 22(9): 1239-1242.

Taber, K. H., Hayman, L. A., Northrup, S. R., and Maturi, L. (1998). Vital sign changes during infant magnetic resonance examinations. Journal of Magnetic Resonance Imaging, 8(6): 1252-1256.

U.S. Food and Drug Administration, Center for Devices and Radiological Health. Guidance for industry and FDA staff: Criteria for significant risk investigations of magnetic resonance diagnostic devices. July 14, 2003. Available at: http://www.fda.gov/cdrh/ode/guidance/793.html. Last accessed February 23, 2009.

U.S. Food and Drug Administration, Center for Drug Evaluation and Research. Information for health care professionals: Gadolinium-based contrast agents for magnetic resonance imaging (marketed as Magnevist, Multi- Hance, Omniscan, OptiMARK, ProHance). June 4, 2008. Available at: http://fda.gov/cder/drug/InfoSheets/HCP/gcca_200705.htm. Last accessed February 23, 2009.

Whitby, E. H., Griffiths, P. D., Lonneker-Lammers, T., Srinivasan, R., Connolly, D. J. A., Capener, D., and Paley, M. N. J. (2004). Ultrafast magnetic resonance imaging of the neonate in a magnetic resonance-compatible incubator with a built-in coil. Pediatrics, 113(2): e150-e152.

Williams, L.-A., DeVito, T. J., Winter, J. D., Orr, T. N., Thompson, R. T., and Gelman, N. (2007). Optimization of 3D MP-RAGE for neonatal brain imaging at 3.0 T. Magnetic Resonance Imaging, 25(8): 1162-1170.

Yang, M., Christoforidis, G., Abduljali, A., and Beversdorf, D. (2006). Vital signs investigation in subjects undergoing MR imaging at 8T. AJNR American Journal of Neuroradiology, 27(4): 922-928. 